\title{
Comparison of electromyographic activity during Nordic hamstring exercise and exercise in lengthened position
}

\author{
Jan Marušič (1), Nejc Šarabon (1,2) \\ (1) University of Primorska, Faculty of Health Sciences, Izola, Slovenia;(2) S2P, Science to \\ practice, Ltd., Laboratory for Motor Control and Motor Behavior, Ljubljana, Slovenia \\ This article is distributed under the terms of the Creative Commons Attribution Noncommercial License (CC BY-NC 4.0) which permits \\ any noncommercial use, distribution, and reproduction in any medium, provided the original author(s) and source are credited.
}

\begin{abstract}
Hamstring strain injuries remain among the most problematic and most frequent sport injuries. Two of the most effective methods for prevention and rehabilitation of the hamstring strain injuries are: classic eccentric training using the Nordic hamstring exercise (NHE) and eccentric training in a lengthened position using the glider exercise. Both exercises have disadvantages that could be fixed by adding hip flexion during the NHE. Thus, the purpose of the study was to compare peak hamstring activity (measured by electromyography) between three eccentric exercises: the standard NHE, the modified NHE and the glider. Differences were statistically tested with the analysis of the variance for repeated measurements and the paired 2-tailed posthoc t-test. Hamstring activity during the modified NHE was significantly lower compared to the NHE and significantly higher compared to the glider. The results indicate that implementing the modified NHE could increase the effectiveness of already established rehabilitation protocols and help reduce the risk of hamstring (re-)injury.
\end{abstract}

Key Words: electromyography, biceps femoris, eccentric training, prevention, rehabilitation

Incidence of hamstring strain injuries (HSI) and reinjuries is still high or even increasing, despite extensive research in the last decades on prevention, rehabilitation, risk factors and injury mechanisms for the occurrence of these injuries. ${ }^{1}$ They are frequent in high-speed running sports, such as track and field, soccer and Gaelic football. $^{2-4}$ Athletes are most often injured during sprinting in the final swing phase, ${ }^{5}$ in which hamstrings are highly active and stretched by the knee extension and hip flexion. ${ }^{6}$ Prevention and rehabilitation protocols often consist of eccentric training with the Nordic hamstring exercise (NHE), which has proven to be one of the most effective ways of preventing HSI. ${ }^{7}$ Knee flexors weakness and short fascicle length of the biceps femoris $(\mathrm{BF}),{ }^{8}$ which is the most frequently injured hamstring muscle,${ }^{9}$ are two large, but modifiable injury risk factors. NHE interventions positively affect these two factors by increasing knee flexors strength, ${ }^{10}$ and length of BF fascicles. ${ }^{11}$ Furthermore, studies show positive effects of hamstring eccentric training in lengthened position on prevention and rehabilitation for HSI, ${ }^{12,13}$ although the mechanisms behind it is yet unknown. The glider from Askling L-protocol is an example of eccentric exercise in lengthened position of the hamstrings, unlike the NHE, in which the neutral hip position does not allow loading at longer hamstring lengths. As a result, hamstrings' lengths are relatively short, especially for individuals who are unable to perform the exercise in a controlled manner throughout the full range of motion. However, the glider enables the athlete to achieve eccentric

\begin{tabular}{ccc}
\multicolumn{2}{c}{ Table 1. Advantages and disadvantages of the Nordic hamstring exercise and the glider } \\
\hline Exercise & Advantages & Disadvantages \\
\hline $\begin{array}{c}\text { The Nordic hamstring } \\
\text { exercise }\end{array}$ & high hamstring activity & $\begin{array}{c}14,15 \\
\text { shorter hamstring lengths during } \\
\text { eccentric loading }\end{array}$ \\
\hline The glider & $\begin{array}{c}\text { eccentric loading in lengthened position of } \\
\text { the hamstrings - similar to the last swing } \\
\text { phase of sprinting }\end{array}$ & $\begin{array}{c}\text { low hamstring activity, } 40 \% \text { of } \\
\text { maximal voluntary contraction }\end{array}$ \\
\hline
\end{tabular}


hamstring loading in positions that are comparable to those in the final swing phase, in which most HSI occur. The main advantages and disadvantages of both exercises are shown in Table 1. Both disadvantages of the exercises could be theoretically improved with the modified NHE (described under Methods). To our knowledge, it has not yet been verified how the peak muscle activity compares between the glider, the NHE and the modified NHE. Thus, the purpose of the study was to compare muscle activity of $\mathrm{BF}, m$. semitendinosus (ST) m. gastrocnemius (GA), m. erector spinae (ES) and $m$. gluteus maximus (GM) during these three exercises. We hypothesized that the peak muscle activity of all measured muscles would be greatest during the NHE and that the peak activity during the modified NHE would be significantly greater than during the glider

\section{Materials and Methods}

\section{Participants}

33 participants volunteered for the study (mean \pm standard deviation): age $27 \pm 2.5$ years; height $178.6 \pm$ $8.1 \mathrm{~cm}$; weight $74.4 \pm 14.5 \mathrm{~kg}$; body mass index $23.1 \pm$ $3.3 \mathrm{~kg} / \mathrm{m}^{2}$; body fat $15.8 \pm 5.9 \%$. All participants were informed about the purpose and content of the study and gave written informed consent prior to participation. The study was approved by the National Medical Ethics Committee (0120-690/2017/8) and conducted according to the Declaration of Helsinki. Inclusion criteria: absence of injuries and illness at the time of measurements, moderate physical activity, ability to perform the NHE in a controlled manner to a minimum of $50 \%$ of total range of motion. Exclusion criteria: neural, muscular, skeletal or connective tissue injuries during the last year in the area of the back, hips and/or legs.

\section{Exercises}

The glider and the modified NHE are both eccentric exercises for the hamstrings in a lengthened position (Figure 1). The modified NHE, which has already been presented and analysed for the purpose of optimizing the standard NHE in two actual studies, ${ }^{17,18}$ is eccentric knee flexion exercise during which the participant maintains increased hip flexion. Participants in our study positioned themselves in $\sim 75^{\circ}$ hip flexion (monitored by the assistant with a goniometer) prior to the descend and were verbally encouraged to maintain hip flexion during the descend. If there were greater (visible) deviations in hip position, then the trial was repeated. The $75^{\circ}$ angle was chosen based on previous study. ${ }^{18}$ The glider is eccentric hip extension exercise, during which the participant maintains almost full knee extension.

\section{Study protocol}

Participants' body composition was measured using bioelectrical impedance analysis (MC-980MA, Tanita Europe B.V., Amsterdam, The Netherlands). Locations for the placement of electromyographic (EMG) sensors were identified and prepared according to Surface ElectroMyoGraphy for the Non-Invasive Assessment of Muscles (SENIAM) recommendations. ${ }^{19}$ EMG sensors were fitted on the long head of BF, ST, lateral head of GA and GM of the dominant leg and on the ER (same side). General, whole-body warm up consisted of 6-min stepping on a $25 \mathrm{~cm}$ high box and 1 set of 10 repetitions of the following bodyweight exercises: squats, heel rises, hip bridges, Jackknife sit-ups and hip extensions. The subjects first performed two 3-sec long maximal voluntary contractions (MVC) for the purpose of EMG signal normalisation: for $\mathrm{BF}$ and $\mathrm{ST}$, participants did knee flexion at $45^{\circ}$ against fixed straps, placed above the malleolus, while lying in a prone position on a physio bed; for GA, participants did plantar flexion against external resistance in neutral ankle position in an upright stance; for GM, participants did hip extension in a prone position on a physio bed against fixed straps, placed just above the knee, with $90^{\circ}$ knee flexion; for ER, participants performed trunk extension in isometric dynamometer (S2P, Science to practice, Ltd., Ljubljana, Slovenia) in an upright stance with fixed pelvis. Subsequently, a series with three repetitions were performed for each exercise (the NHE, the modified

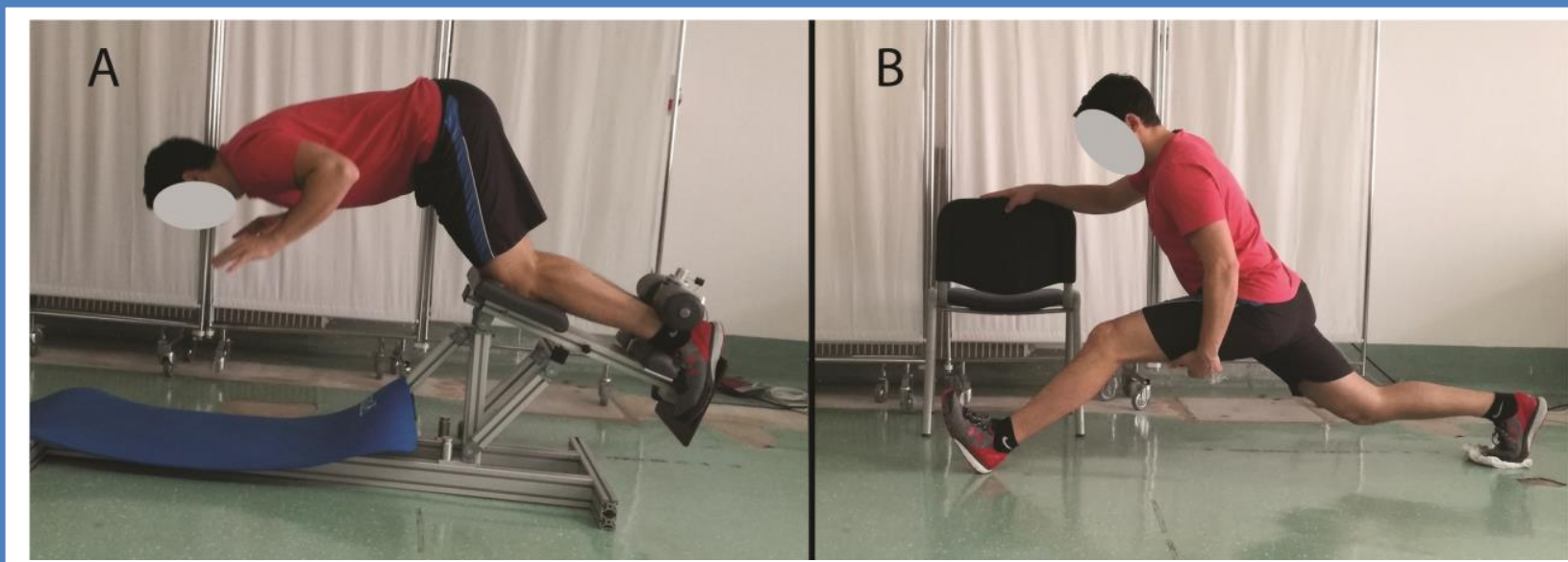

Fig 1. The modified Nordic hamstring exercise (A) and the glider (B). 


\section{EMG during eccentric hamstring exercises}

Eur J Transl Myol 30 (2): 234-239, 2020

NHE and the glider) in a randomized order. The rest between each repetition lasted 5-10 sec, the rest between the individual exercises was set to $3 \mathrm{~min}$. Loud verbal encouragement was provided during all trials. Prior to the measured set, participants did a familiarization set of 3 repetitions for each exercise.

\section{Data acquisition and processing}

Trigno Delysis measuring system with pre-amplified self-adhesive wireless electrodes $(27 \times 37$ x $15 \mathrm{~mm}$; weight: $14.7 \mathrm{~g}$ ) and EMGWorks software (Delsys Inc., Massachusetts, USA) were used to capture electromyographic signals at a frequency of $2000 \mathrm{~Hz}$. Signals were analysed in the following order: 1) band pass filtration using Butterworth second-order filter (20$500 \mathrm{~Hz}), 2$ ) rectification, using root mean square function (0.05 second window length and point-by-point overlap), 3) smoothing, using moving average function (0.05 second window length and point-by-point overlap).
Highest mean value on 0.25 window length was then calculated as peak muscle activity and normalised with peak activity during MVC trials for each muscle.

\section{Statistical analysis}

For the statistical analysis, the SPSS 25 program (IBM, New York, USA) was used. Descriptive statistics were calculated and reported as mean \pm standard deviation. Shapiro-Wilk test was used for testing of normality and Levene's test for equality of variances. Differences between exercises were analysed by analysis of variance for repeated measurements. Effect sizes were calculated and interpreted as small $\left(\eta^{2}=0.01\right)$, medium $\left(\eta^{2}=0.06\right)$ and large $\left(\eta^{2}=0.14\right) .{ }^{20}$ For pair-wise comparisons, paired 2-tailed post-hoc t-tests with Bonferroni's correction were used. Intra-session repeatability was assessed by calculating typical error of measurement, coefficient of variance and two types of intraclass correlation coefficients (ICC) (model 2,1 and 2,k). Reliability was

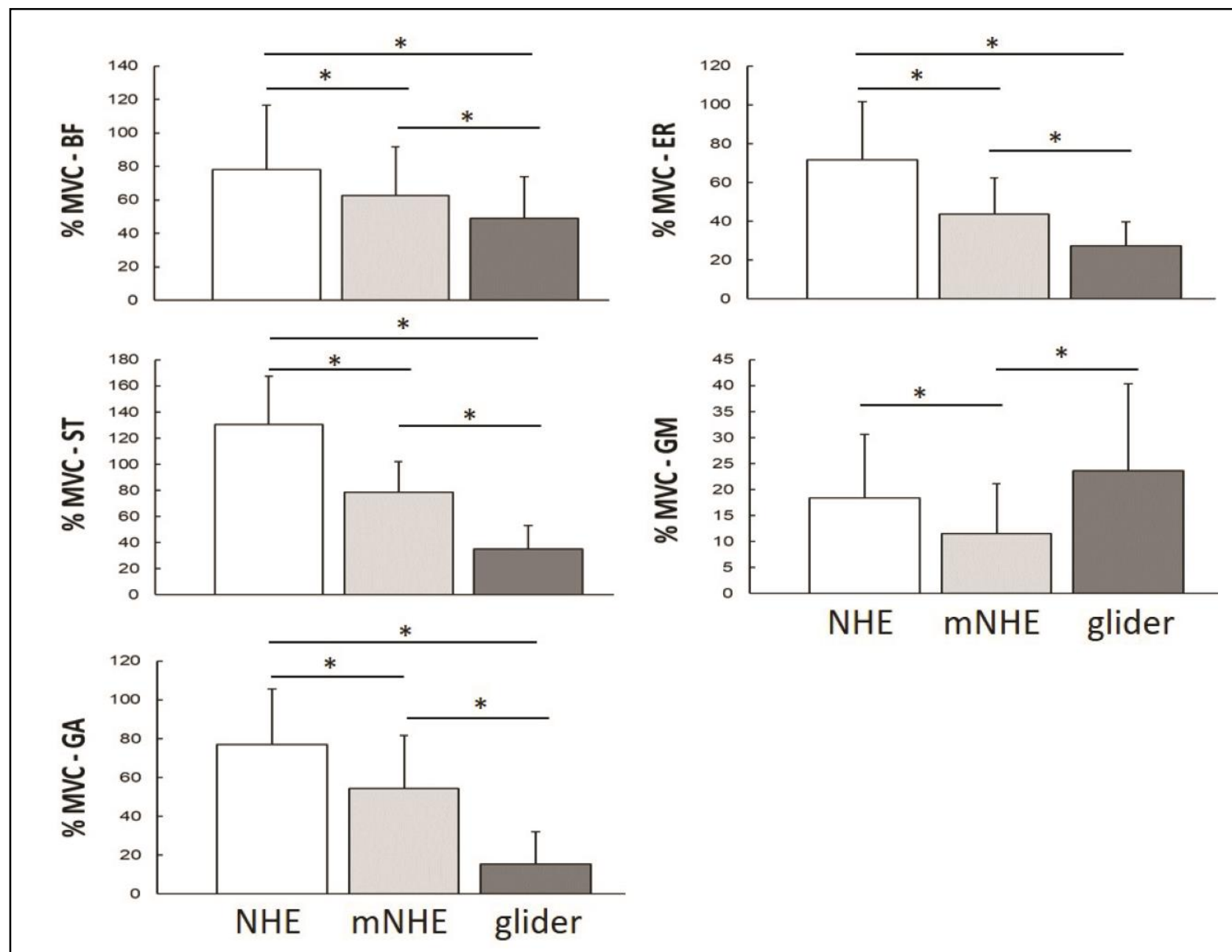

Fig 2. Differences in peak electromyographic activity.

$\mathrm{BF}$ - biceps femoris, ST - semitendinosus, GA - gastrocnemius, ES - erector spinae, GM - gluteus maximus, \% MVC - \% of maximal voluntary contraction, NHE - Nordic hamstring exercise, mNHE - modified Nordic hamstring exercise 
interpreted as poor $(<0.5)$, moderate (between 0.5 and 0.75), good (between 0.75 and 0.9) and excellent $(>0.9) .{ }^{20}$ The level of statistical significance was set at $\mathrm{p}$ $<0.05$.

\section{Results}

The subjects successfully and technically correctly performed all repetitions for which EMG signals were captured. Further manual inspection revealed $6.5 \%$ of the signals that were unsuitable for entering the statistical analysis. Peak muscle activity was significantly different between exercises for all measured muscles $\left(\mathrm{F}_{(1,3-2,0)}=\right.$ $\left.15,0-105,8 ; \mathrm{p}<0,001 ; \eta^{2}=0,34-0,82\right)$. The pairwise comparisons between exercises revealed significant difference for all measured muscles ( $p<0.001)$ except for the GM between the NHE and the glider $(\mathrm{p}=0.09)$ (Figure 2). Peak muscle activity significantly decreased for all measured muscles in the following order: the NHE $>$ the modified NHE > the glider. Intra-session repeatability was good to excellent for all tasks and measured muscles $\left(\mathrm{ICC}_{2, \mathrm{k}}=0.91-0.99 ; \mathrm{ICC}_{2,1}=0.78\right.$ 0.97) (Table 2).

\section{Discussion}

The purpose of the study was to check the peak muscle activity during the two of the most effective exercises (the NHE and the glider) for prevention and rehabilitation of the HSI. Additionally, the purpose was to test whether the hip flexion adjustment of the NHE could achieve significantly higher hamstring peak muscle activity compared to the glider while maintaining eccentric loading in lengthened position. The results of the study confirm our hypotheses about the significantly different activities for all measured muscles except for GM: the participants achieved the highest peak muscle activity during the NHE, significantly lower peak during the modified NHE and the lowest peak during the glider. GM activity was greatest during the glider, probably due to specific technique, as it is a hip dominant exercise, in which GM has an eccentric function compared to the isometric hip maintenance function during the knee dominant NHE or modified NHE. Hamstring peak muscle activity was significantly higher during the NHE compared to the modified version, which is in line with the results of previous studies. ${ }^{18,21}$ Moreover, both exercises produced higher peak activity for ST compared to $\mathrm{BF}$, which is consistent with the results of previous study. ${ }^{22}$ Movement across the knee joint appears to elicit greater ST peak activity compared to hip movement, during which is BF peak activity relatively higher, ${ }^{15}$ which is also confirmed by our results (the knee dominant modified NHE compared to the hip dominant glider). Furthermore, peak hamstring activity during the modified NHE was significantly higher than during the glider, during which the highest peak activity was $37.3 \pm$ $19.2 \% \mathrm{MVC}$ for BF and $34.9 \pm 18.1 \% \mathrm{MVC}$ for ST, which is in line with the results ( $40 \%$ MVC) of previous research on peak muscle activity during L-protocol

Table 2. Intra-session repeatability of normalised peak electromyographic activity, mean (SD).

\begin{tabular}{|c|c|c|c|c|c|c|c|c|}
\hline \multicolumn{2}{|c|}{ Task/muscle } & $\operatorname{Rep} 1$ & $\operatorname{Rep} 2$ & $\operatorname{Rep} 3$ & TEM & $\% \mathrm{CV}$ & $\mathrm{ICC}_{2,1}$ & $\mathrm{ICC}_{2, \mathrm{k}}$ \\
\hline \multirow{5}{*}{ 荣 } & GA & $75.2(34.2)$ & $82.0(39.3)$ & $83.4(44.4)$ & 6.62 & 8.3 & 0.90 & 0.96 \\
\hline & ST & $125.7(35.0)$ & $133.3(40.4)$ & $132.0(37.3)$ & 2.77 & 2.1 & 0.94 & 0.98 \\
\hline & ES & $19.1(14.3)$ & $19.6(14.6)$ & $20.5(18.0)$ & 2.35 & 11.9 & 0.92 & 0.97 \\
\hline & GM & $67.5(32.4)$ & $73.8(33.2)$ & $76.1(29.2)$ & 9.50 & 13.1 & 0.83 & 0.94 \\
\hline & BF & $113.9(38.2)$ & $122.1(36.5)$ & $124.4(36.5)$ & 5.27 & 4.4 & 0.90 & 0.97 \\
\hline \multirow{5}{*}{$\begin{array}{l}\mathbf{z} \\
\text { 音 }\end{array}$} & GA & $50.0(27.3)$ & $53.7(26.5)$ & $59.4(31.2)$ & 4.88 & 9.0 & 0.89 & 0.96 \\
\hline & ST & $77.3(24.2)$ & $77.5(26.8)$ & $81.4(19.5)$ & 5.31 & 6.7 & 0.82 & 0.93 \\
\hline & ES & $11.2(10.4)$ & $11.5(9.7)$ & $11.7(9.8)$ & 0.73 & 6.3 & 0.97 & 0.99 \\
\hline & GM & $43.0(19.5)$ & $45.6(22.2)$ & $48.8(22.6)$ & 5.86 & 12.8 & 0.86 & 0.95 \\
\hline & BF & $69.6(29.9)$ & $71.1(28.1)$ & $77.5(25.6)$ & 3.63 & 5.0 & 0.89 & 0.96 \\
\hline \multirow{5}{*}{ 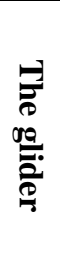 } & GA & $13.4(14.2)$ & $16.6(20.0)$ & $17.3(18.2)$ & 3.97 & 25.1 & 0.89 & 0.96 \\
\hline & ST & $35.6(18.2)$ & 34.4 (18.4) & $37.0(19.6)$ & 3.53 & 9.9 & 0.87 & 0.95 \\
\hline & ES & $27.6(21.7)$ & $26.1(21.5)$ & $28.8(24.0)$ & 2.82 & 10.3 & 0.94 & 0.98 \\
\hline & GM & $26.3(13.0)$ & $26.5(12.8)$ & $28.3(13.6)$ & 3.04 & 11.3 & 0.85 & 0.95 \\
\hline & BF & $37.4(19.9)$ & $38.2(23.9)$ & $37.3(18.9)$ & 8.39 & 22.3 & 0.78 & 0.91 \\
\hline
\end{tabular}


exercises. ${ }^{16}$ The modified NHE elicited significantly higher peak hamstring activity during eccentric loading $(72.4 \pm 27.2 \% \mathrm{MVC}$ for $\mathrm{BF}$ and $78.5 \pm 23.6 \% \mathrm{MVC}$ for ST) at a similarly lengthened hamstring position compared to the glider. The latter suggests that the proper implementation of the modified NHE could increase the effectiveness of existing rehabilitation or prevention protocols for the HSI in terms of greater positive adaptations to the architectural and/or functional hamstring characteristics, which are relevant in preventing HSI. Theoretically, an eccentric loading in lengthened position with sufficient muscle activity would more effectively elongate muscle fascicles, which is one of the key mechanisms for reducing the risk of HSI occurrence. Furthermore, achieving a similarly stretched hamstring position during eccentric exercise as occurs in the last swing phase during high-speed running could have additional positive effects on HSI prevention. To our knowledge, only one study so far has compared the effects of eccentric training in a lengthened position (eccentric knee flexion on an isokinetic dynamometer with $90^{\circ}$ hip flexion - seated position) with eccentric training at short hamstring lengths (eccentric knee flexion on an isokinetic dynamometer with a hip in a neutral position - supine) on the functional and architectural characteristics of the hamstrings. ${ }^{23}$ There were no significant differences between the groups, however the intervention lasted only 3 weeks. The authors concluded that a longer intervention would show significantly different increases in muscle fascicles with greater effect sizes when using eccentric training in a lengthened position. Further research using practical exercises (e.g. the modified NHE or the glider) and longer training interventions is needed to verify these assumptions and to discover the mechanisms behind hamstring eccentric training in a lengthened position that cause preventive effects. Good and excellent intrasession repeatability shown in our study is comparable to previous study, in which authors analysed normalised peak hamstring activity during the NHE. ${ }^{24}$ The main limitation of the study is the use of EMG method in combination with only one measurement set for each exercise. Surface EMG can be prone to cross-talk from nearby muscles, especially between ST and semimembranosus. Additionally, normalisation technique can also affect the results from hamstring muscles, as it is still not clear whether the knee flexion MVC can elicit similar activity from both BFlh and ST. In conclusion, the glider elicits relatively low peak hamstring activity compared to the NHE, but enables eccentric loading in a lengthened position of the hamstring similar to those that occur during last swing phase of high-speed running, i.e. the moment when most HSI occur. The modified NHE could be a logical upgrade for the NHE and the glider, as it combines the benefits of both exercises: relatively high hamstring activity and eccentric loading in a lengthened position in a practical way, since it requires no special equipment.

\section{List of acronyms}

$\mathrm{BF}$ - biceps femoris,

ES - erector spinae,

GA - gastrocnemius,

GM - gluteus maximus

mNHE - modified Nordic hamstring exercise

MVC - maximal voluntary contraction,

NHE - Nordic hamstring exercise,

SENIAM - Surface ElectroMyoGraphy for the Non-

Invasive Assessment of Muscles

ST - semitendinosus,

\section{Authors contributions}

NŠ conceptualized the study and finalized the paper. JM carried out the measurements, analysed data and wrote the manuscript draft.

\section{Acknowledgments}

None.

\section{Funding}

The study was supported by the Slovenian Research Agency through the project TELASI-PREVENT [L51845] (Body asymmetries as a risk factor in musculoskeletal injury development: studying aetiological mechanisms and designing corrective interventions for primary and tertiary preventive care).

\section{Conflict of Interest}

The authors declare that they have no known competing financial interests or personal relationships that could have appeared to influence the work reported in this paper.

\section{Ethical Publication Statement}

We confirm that we have read the Journal's position on issues involved in ethical publication and affirm that this report is consistent with those guidelines.

\section{Corresponding Author}

Nejc Šarabon, Faculty of Health Sciences, University of Primorska, Polje 42, SI-6310 Izola, Slovenia.

Tel. +38656626466

ORCID iD: 0000-0003-0747-3735

E-mail: nejc.sarabon@fvz.upr.si

\section{Co-author}

Jan Marušič: jan.marusic@fvz.upr.si ORCID iD: 0000-0001-9547-3748

\section{References}

1. Ekstrand J, Waldén M, Hägglund M. Hamstring injuries have increased by $4 \%$ annually in men's professional football, since 2001: A 13-year longitudinal analysis of the UEFA Elite Club injury study. Br J Sports Med 2016;50:731-7.

2. Roe M, Murphy JC, Gissane C, Blake C. Lower limb injuries in men's elite Gaelic football: A prospective investigation among division one teams 


\section{EMG during eccentric hamstring exercises}

Eur J Transl Myol 30 (2): 234-239, 2020

from 2008 to 2015. J Sci Med Sport [Internet] 2018;21:155-9.

3. Opar DA, Drezner J, Shield A, et al. Acute hamstring strain injury in track-and-field athletes: A 3-year observational study at the Penn Relay Carnival. Scand J Med Sci Sports 2014;24:254-9.

4. Ekstrand J, Hägglund $\mathrm{M}$, Waldén $\mathrm{M}$. Injury incidence and injury patterns in professional football: The UEFA injury study. Br J Sports Med 2011;45:553-8.

5. Brooks JHM, Fuller CW, Kemp SPT, Reddin DB. Incidence, risk, and prevention of hamstring muscle injuries in professional rugby union. Am J Sports Med 2006;34:1297-306.

6. Chumanov ES, Heiderscheit BC, Thelen DG. Hamstring musculotendon dynamics during stance and swing phases of high-speed running. Med Sci Sports Exerc 2011;43:525-32.

7. Al Attar WSA, Soomro N, Sinclair PJ, et al. Effect of Injury Prevention Programs that Include the Nordic Hamstring Exercise on Hamstring Injury Rates in Soccer Players: A Systematic Review and Meta-Analysis. Sport Med 2017;47:907-16.

8. Timmins RG, Bourne MN, Shield AJ, et al. Short biceps femoris fascicles and eccentric knee flexor weakness increase the risk of hamstring injury in elite football (soccer): A prospective cohort study. Br J Sports Med 2016;50:1524-35.

9. Koulouris G, Connell DA, Brukner P, SchneiderKolsky M. Magnetic resonance imaging parameters for assessing risk of recurrent hamstring injuries in elite athletes. Am J Sports Med 2007;35:1500-6.

10. Delahunt E, McGroarty M, De Vito G, Ditroilo M. Nordic hamstring exercise training alters knee joint kinematics and hamstring activation patterns in young men. Eur J Appl Physiol 2016;116:663-72.

11. Alonso-Fernandez, D., Docampo-Blanco P, Martinez-Fernandez J. Changes in muscle architecture of biceps femoris induced by eccentric strength training with nordic hamstring exercise. Scand J Med Sci Sports 2018;28:88-94.

12. Askling $\mathrm{C}$, Tengvar $\mathrm{M}$, Tarassova $\mathrm{O}$, Thorstensson A. Acute hamstring injuries in Swedish elite sprinters and jumpers: A prospective randomised controlled clinical trial comparing two rehabilitation protocols. $\mathrm{Br} \mathrm{J}$ Sports Med 2014;48:532-9.

13. Askling $\mathrm{C}$, Tengvar $\mathrm{M}$, Thorstensson A. Acute hamstring injuries in Swedish elite football: a prospective randomised controlled clinical trial comparing two rehabilitation protocols. Br J Sports Med 2013;47:953-9.

14. Zebis MK, Skotte J, Andersen CH, et al. Kettlebell swing targets semitendinosus and supine leg curl targets biceps femoris: An EMG study with rehabilitation implications. $\mathrm{Br} \mathrm{J}$ Sports Med 2013;47:1192-8.

15. Bourne MN, Duhig SJ, Timmins RG, et al. Impact of the Nordic hamstring and hip extension exercises on hamstring architecture and morphology: Implications for injury prevention. Br J Sports Med 2017;51:469-77.

16. Severini G, Holland D, Drumgoole A, et al. Kinematic and electromyographic analysis of the Askling L-Protocol for hamstring training. Scand J Med Sci Sport 2018;28:2536-46.

17. Giacomo J-P, Lahti J, Hegyi A, et al. A new testing and training device for hamstring muscle function. Sport Performance Sci Reports 2018;40:1-4. 18.

18. Šarabon N, Marušič J, Marković G, Kozinc Ž. Kinematic and electromyographic analysis of variations in Nordic hamstring exercise. PLoS One 2019;14:1-16.

19. Hermens HJ, Freriks B, Merletti R, et al. European recommendations for surface electromyography. Roessingh Res Dev 1999;8:13-54.

20. Cohen J. Statistical Power Analysis for the Behavioral Sciences. Abingdon: Routledge; 1988.

21. Hegyi A, Lahti J, Giacomo JP, et al. Impact of hip flexion angle on unilateral and bilateral nordic hamstring exercise torque and high- density electromyography activity. J Orthop Sports Phys Ther 2019;49:584-92.

22. Bourne MN, Opar DA, Williams MD, et al. Muscle activation patterns in the Nordic hamstring exercise: Impact of prior strain injury. Scand J Med Sci Sport 2016;26:666-74.

23. Guex K, Degache F, Morisod C, et al. Hamstring architectural and functional adaptations following long vs. short muscle length eccentric training. Front Physiol 2016:1-9.

24. Ditroilo M, De Vito G, Delahunt E. Kinematic and electromyographic analysis of the Nordic hamstring exercise. J Electromyogr Kinesiol 2013;23:1111-8.

Submission: March 10, 2020

Revision received: March 26, 2020 Accepted for publication: March 26, 2020 